\title{
gु \\ Electronic structure near an impurity and terrace on the surface of a three-dimensional topological insulator
}

\author{
Qiang-Hua Wang, ${ }^{1, *}$ Da Wang, ${ }^{1}$ and Fu-Chun Zhang ${ }^{2}$ \\ ${ }^{1}$ National Laboratory of Solid State Microstructures and Department of Physics, Nanjing University, Nanjing 210093, China \\ ${ }^{2}$ Department of Physics, The University of Hong Kong, Pokfulam Road, Hong Kong, China
}

(Received 22 October 2009; published 11 January 2010)

\begin{abstract}
Motivated by recent scanning tunneling microscopy experiments on surfaces of $\mathrm{Bi}_{1-x} \mathrm{Sb}_{x^{\prime}}$ and $\mathrm{Bi}_{2} \mathrm{Te}_{3}$, we theoretically study the electronic structure of a three-dimensional (3D) topological insulator in the presence of a local impurity or a domain wall on its surface using a 3D lattice model. While the local density of states (LDOS) oscillates significantly in space at energies above the bulk gap, the oscillation due to the in-gap surface Dirac fermions is very weak. The extracted modulation wave number as a function of energy satisfies the Dirac dispersion for in-gap energies and follows the border of the bulk continuum above the bulk gap. We have also examined analytically the effects of the defects by using a pure Dirac fermion model for the surface states and found that the LDOS decays asymptotically faster at least by a factor of $1 / r$ than that in normal metals, consistent with the results obtained from our lattice model.
\end{abstract}

DOI: 10.1103/PhysRevB.81.035104

PACS number(s): 73.20.At, 73.20.Hb, 73.90.+f

\section{INTRODUCTION}

A three-dimensional (3D) topological insulator (TI) is a time-reversal invariant system with bulk gap but supports massless Dirac fermions with coupled spin and momentum on the surface. ${ }^{1,2}$ The existence and the (odd) number of Dirac cones of the massless dispersion are protected by the $Z_{2}$ topology of the bulk band structure. ${ }^{3}$ Specific materials of 3D topological insulators have recently been studied both experimentally ${ }^{4}$ and theoretically. ${ }^{5}$ Before harvesting the novel properties of TIs, ${ }^{6}$ an interesting question is how robust the surface Dirac fermions are against imperfections, such as local and extended impurities. This issue is made interesting and challenging by recent scanning tunneling microscopy (STM) measurements. ${ }^{7-10}$ It is observed that while local impurities are seen to induce local density of states (LDOS) oscillation, ${ }^{9}$ surprisingly the domain wall (a terrace on the surface) does not seem to induce LDOS oscillations for energies within the bulk gap. ${ }^{7,8,10}$

We investigate the above issue by a model lattice Hamiltonian for a three-dimensional TI. The main results are as follows. (1) We obtain the surface Green's function and therefore the spectral function for the surface states. The contributions from surface Dirac fermions and the bulk extended states are clearly identified. (2) For an upper terrace on the surface, we find the LDOS oscillation is vanishingly weak for energies below the bulk gap $\Delta$, where Dirac fermions are best defined. The oscillation is significant above $\Delta$, but it can be clearly ascribed to the contributions from the bulk extended states near the bottom of the conduction band. The numerical phenomenology is in nice agreement with the experiment. ${ }^{7,8,10}$ On the other hand, the LDOS oscillation on the lower terrace is globally weak. The asymmetry of the terraces can be attributed to the difference in the effective scatting mechanism. (3) For a local unitary impurity on a flat surface, we find LDOS oscillations at all energies, although it is relatively weaker below $\Delta$. (4) Combining both terraces and local impurities, we extract from the LDOS oscillations the energy $\omega$ dependence of the modulation wave number
$2 k_{\omega}$. For $\omega<\Delta$, the dispersion ( $\omega$ vs. $k_{\omega}$ ) coincides with that of surface Dirac fermions, while for $\omega>\Delta$ it is actually related to the bulk extended states. The above numerical results combine to support the robustness of surface Dirac fermions. (5) For comparison, we substantiate analytical results using pure Dirac fermion models subject to impurity scattering. Asymptotically, the oscillation in LDOS, if present at all, has an energy-dependent wave number $2 k_{\omega}$, where $k_{\omega}$ is the onshell momentum, and decays faster by a factor of $1 / r$ than that for usual fermions. In particular, a hard domain wall in two-dimensional (2D) Dirac models does not lead to any LDOS oscillations at all. These are in qualitative agreement with the numerical results for the full 3D model. However, we do find and discuss differences between surface and pure Dirac fermions in connection to the nature of the wave functions.

The rest of the paper is organized as follows. We discuss the surface states using a 3D lattice model in Secs. II-IV, where a perfect surface, a terrace, and a local impurity are discussed, respectively. Section V contains analytical results using pure Dirac models. We summarize and provide remarks in connection to experiments in Sec. VI.

\section{SURFACE STATES IN A LATTICE MODEL OF TOPOLOGICAL INSULATORS}

We start with a 3D lattice model for topological insulators. The Hamiltonian is given by $H=\Sigma_{\mathbf{k}} \psi_{\mathbf{k}}^{\dagger} h_{\mathbf{k}} \psi_{\mathbf{k}}$, where $\psi_{\mathbf{k}}$ is a four-spinor, $\mathbf{k}$ is the lattice momentum, and

$$
h_{\mathbf{k}}=\left[m-\sum_{b} 2 t_{b}\left(\cos k_{b}-1\right)\right] \Gamma_{0}+\sum_{b} 2 t_{b} \sin k_{b} \Gamma_{b},
$$

where $m$ is a parameter controlling the bulk gap, $t_{b}$ is the hopping amplitude along a bond $\mathbf{b}$ (see below), $k_{b}=\mathbf{k} \cdot \mathbf{b}$, and $\Gamma_{b}=\left(\Gamma_{1} \hat{x}+\Gamma_{2} \hat{y}+\Gamma_{3} \hat{z}\right) \hat{b}$. Here, $\hat{x}, \hat{y}$, and $\hat{z}$ are three orthogonal unit vectors and $\hat{b}=\mathbf{b} / b$. Furthermore, $\Gamma_{0,1,2,3}$ are Dirac matrices that satisfy the Clifford algebra $\left\{\Gamma_{\mu}, \Gamma_{\nu}\right\}=2 \delta_{\mu \nu}$. Explic- 
(a)

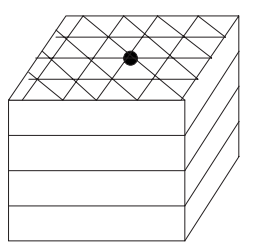

(b)

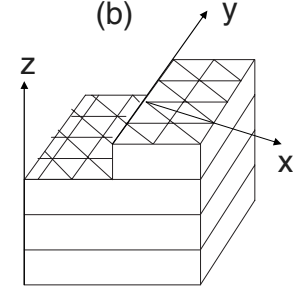

FIG. 1. Schematic plot of a topological insulator with layered hexagonal structure and (a) a local impurity (filled circle) and (b) (upper and lower) terraces on the surface. Here, $x, y$, and $z$ denote the orthogonal axes and the normal direction of the terrace edge is along $x$. The interlayer hopping is along the vertical direction $z$.

itly, we take $\Gamma_{1}=\sigma_{1} \otimes \tau_{3}, \Gamma_{2}=\sigma_{2} \otimes \tau_{3}, \Gamma_{3}=\sigma_{0} \otimes \tau_{2}$, and $\Gamma_{0}$ $=\sigma_{0} \otimes \tau_{1}$, where $\sigma$ and $\tau$ are Pauli matrices $\left(\sigma_{0}\right.$ is the unit matrix). However, the general conclusion does not rely on the parameterization of the Dirac matrices. In order to respect the sixfold symmetry of the samples in the experiments, ${ }^{7-10}$ we consider a nearest-neighbor tightbinding model illustrated in Fig. 1 (impurities are considered in the next section). The structure contains in-plane hexagonal layers and stacked vertically in an $A A \ldots$ fashion. We set all lattice constants to be unity and therefore the nearestneighbor bond vectors are specified by $\mathbf{b}=(1,0,0)$ and $(1 / 2, \pm \sqrt{3} / 2,0)$ for in-plane hopping and $\mathbf{b}=(0,0,1)$ for interlayer hopping. (Notice that $-\mathbf{b}$ is also a bond for hopping but has already been counted in the dispersion in the above Hamiltonian.) Decomposing $h_{\mathbf{k}}$ as $h_{\mathbf{k}}=\Sigma_{\mu} \xi_{\mathbf{k}, \mu} \Gamma_{\mu}$, one sees that the bulk dispersion is given by $\omega= \pm E_{\mathbf{k}}$ with $E_{\mathbf{k}}$ $=\sqrt{\sum_{\mu} \xi_{\mathbf{k}, \mu}^{2}}$ and the bulk gap is defined by $\Delta=\min E_{\mathbf{k}}$. For simplest purpose, we set $2 t_{\mathbf{b}}=1$ for all $\mathbf{b}$ and $m=1$ henceforth. In this case, $\Delta=1$ and the system is a topological insulator, ${ }^{2}$ since only one Dirac point exits in the entire surface Brillouin zone (see below).

We use a recursion method to obtain the surface Green's function. In the presence of open surfaces normal to $z$, the planar momentum $\mathbf{k}_{\|}=\left(k_{x}, k_{y}\right)$ is still a good quantum number. For each $\mathbf{k}_{\|}$, the Hamiltonian can be decomposed formally into intralayer part $\Sigma_{n} \psi_{n}^{\dagger} h_{\mathbf{k}_{\|}}^{2 d} \psi_{n}$ and interlayer part $\sum_{n} \psi_{n}^{\dagger} h_{z} \psi_{n+1}+$ H.c., where $n$ is the layer index,

$$
h_{\mathbf{k}_{\|}}^{2 \mathrm{D}}=\left[m+2 t_{z}-\sum_{\mathbf{b} \neq \hat{z}} 2 t_{b}\left(\cos k_{b}-1\right)\right] \Gamma_{0}+\sum_{\mathbf{b} \neq \hat{z}} 2 t_{b} \sin k_{b} \Gamma_{b},
$$

and $h_{z}=-t_{z}\left(\Gamma_{0}+i \Gamma_{3}\right)$ before setting our parametrization. Define $g=1 /\left(z \mathbf{I}-h_{\mathbf{k}_{\|}}^{2 \mathrm{D}}\right)$ as the Green's function for an isolated layer (here, $z=\omega+i 0^{+}$for retarded Green's function, and $\mathbf{I}$ is the $4 \times 4$ unit matrix), the surface Green's function for an $\mathrm{N}$-layer lattice is given by, recursively,

$$
G_{N}^{-1}=g^{-1}-h_{z}^{\dagger} G_{N-1} h_{z},
$$

starting with $G_{1}=g$. Amazingly, it is able to synthesize samples with a series of layers in recent experiments. ${ }^{11}$ In this case, the recursion method is particularly useful to reveal the evolution of the surface states as the sample thickness increases. However, in this paper, we are only interested in infinite-layer lattices, for which $G^{-1}=g^{-1}-h_{z}^{\dagger} G h_{z}$ holds for $G=G_{\infty}$.

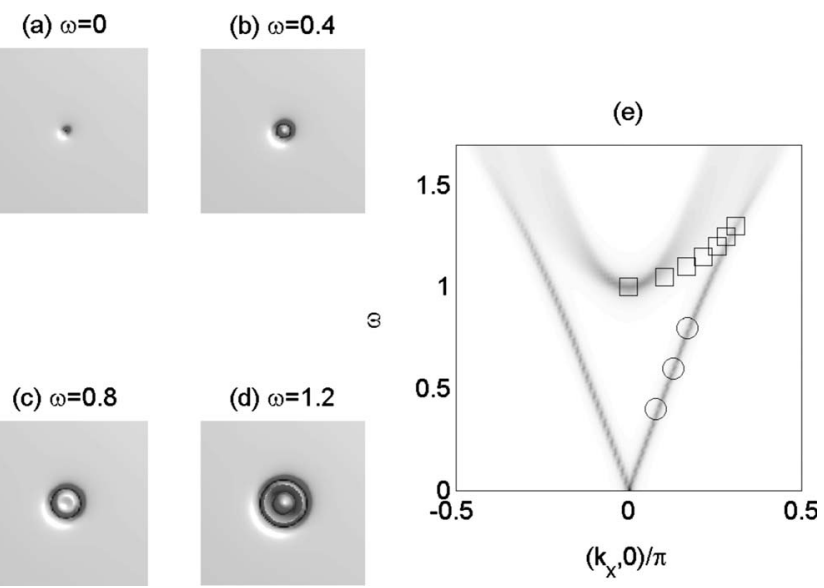

FIG. 2. [(a)-(d)] Intensity plot of $A\left(\mathbf{k}_{\|}, \omega\right)$ in the planar $\mathbf{k}_{\|}$space at $\omega=0,0.4,0.8$, and 1.2. The intensity scales with the darkness. The view field is bounded by $2 \pi \times 2 \pi$ (within the hexagonal Brillouin zone). Camera lighting is used to enhance the contrast. (e) Intensity plot of $A\left(\mathbf{k}_{\|}, \omega\right)$ along a cut $\left(k_{x}, 0\right)$ (gray). Open squares and circles are extracted from LDOS oscillations in Figs. 3(b) and 4 , respectively.

The spectral function for the surface states is given by

$$
A\left(\mathbf{k}_{\|}, \omega\right)=-\frac{1}{\pi} \operatorname{Im} \operatorname{Tr} G_{\mathbf{k}_{\|}},
$$

where the planar momentum is indicated explicitly. The distribution in the momentum space is plot in Figs. 2(a)-2(d) for a series of $\omega$ (only positive energies are displayed since the spectrum is particle-hole symmetric). It is seen that there is only a single dark spot at $\omega=0$. This indicates that we have but one Dirac cone in the Brillouin zone. The spot evolves to a ring with increasing size for $\omega<\Delta=1$. For $\omega>\Delta$, bulk continuum starts to contribute. It is initially a spot at the $\Gamma$ point for $\omega \sim 1$ (not shown) and evolves to a ring close to the Dirac shell quickly at $\omega=1.2$. To see the evolution in energy better, we plot it in Fig. 2(e) (gray) along a line cut $\left(k_{x}, 0\right)$. A clear massless Dirac dispersion is seen. The bulk continuum starts at $\omega=\Delta$ and $\mathbf{k}_{\|}=0$ and the outer border expands with increasing energies, while the Dirac dispersion persists for $\omega>\Delta$ but eventually diminishes for $\omega>1.3$. The contributions from surface Dirac fermions and bulk continuum are clearly separable. In comparison to pure Dirac models, the surface Dirac fermions have a momentum-dependent spectral weight, the exact nature of which will be discussed elsewhere.

\section{TERRACES ON THE SURFACE}

Experimentally, terraces often appear on the surface. We assume the upper terrace is one unit cell higher than the lower one, as in the experiment ${ }^{7-10}$ and illustrated in Fig. 1(b). The Green's function $\mathcal{G}$ on the terraces is obtained as follows. The idea is to obtain the effective theory for the top two layers and treat the terrace situation as a perturbation exactly.

First, for reasons to be clear, we map the hexagonal lattice to a square lattice. This is plausible since both structures 
have one atom per unit cell. Explicitly, we transform b as $(1,0,0) \rightarrow(1,0,0), \quad(1 / 2, \sqrt{3} / 2,0) \rightarrow(1,1,0)$, and $(-1 / 2, \sqrt{3} / 2,0) \rightarrow(0,1,0)$. The hopping amplitudes are not changed and therefore all physical properties are not changed under the structure mapping. Now, each layer is a square lattice, on which we denote the positions in real and momentum space as $(u, v)$ and $(p, q)$, respectively.

Consider a system with $N+2$ layers with $N \rightarrow \infty$. We can integrate out the degrees of freedom in the lower $N$ layers (in the bulk) recursively in a similar fashion to what is described in the previous section, leaving an effective action $S$ with Green's function $G$ for the top two layers

$$
\begin{aligned}
S= & \sum_{\alpha, \beta=L, U} \bar{\psi}_{\alpha}\left(G^{-1}\right)^{\alpha \beta} \psi_{\beta}=\bar{\psi}_{L} g_{s}^{-1} \psi_{L}+\bar{\psi}_{U} g^{-1} \psi_{U} \\
& -\left(\bar{\psi}_{L} h_{z} \psi_{U}+\text { H.c. }\right),
\end{aligned}
$$

where a summation over conserved momenta $(p, q)$ is left implicit, $\alpha$ and $\beta$ are layer indices, and $g_{s}$ is identical to the ideal surface Green's function (for the transformed structure). For later purpose, we define partial Fourier transforms

$$
G_{q}^{\alpha \beta}(u)=\frac{1}{M} \sum_{p} G^{\alpha \beta}(p, q) e^{i p u},
$$

where $M$ is the number of lattice sites along the $u$ or $v$ axis.

Now consider a terrace whose boundary is mapped to the $v$ axis through the origin on the upper layer. The terrace situation is equivalent to setting $m \rightarrow \infty$ in (and only in) the region $\alpha=U$ and $u \leq 0$. Since the terrace is along the $v$ axis, $q$ is still a good quantum number and this convenience motivated our structure mapping. The perturbed Green's function $\mathcal{G}$ can be obtained exactly by the $T$-matrix formalism ${ }^{12}$

$$
\begin{aligned}
\mathcal{G}_{q}^{\alpha \beta}\left(u, u^{\prime}\right)= & G_{q}^{\alpha \beta}\left(u-u^{\prime}\right) \\
& +\sum_{u_{a, b} \leq 0} G_{q}^{\alpha U}\left(u-u_{a}\right) T_{q}\left(u_{a}, u_{b}\right) G_{q}^{U \beta}\left(u_{b}-u^{\prime}\right),
\end{aligned}
$$

where the layer index $U$ and the condition $u_{a, b} \leq 0$ indicate that the depleted region is the upper-left layer and $T_{q}$ is the $q$-resolved $T$ matrix given by $T_{q}^{-1}\left(u_{a}, u_{b}\right)=-G_{q}^{U U}\left(u_{a}-u_{b}\right)$. As what is required, $\mathcal{G}_{q}^{\alpha \beta}\left(u, u^{\prime}\right)=0$ whenever $u \alpha$ or $u^{\prime} \beta$ falls on the upper-left layer. We emphasize that the Green's function obtained this way contains all effects from the bulk below the terraces. The LDOS on the terraces is given by

$$
\rho_{\alpha}(\omega, u)=-\frac{1}{\pi M} \operatorname{Im} \operatorname{Tr} \sum_{q} \mathcal{G}_{q}^{\alpha \alpha}(u, u),
$$

where $\alpha=L(\alpha=U)$ for $u<0(u>0)$. Finally, we map $u$ back to the normal displacement $x$ from the edge on the hexagonal lattice as shown in Fig. 1(b).

The LDOS as a function of $x$ is shown in Figs. 3(a) and 3 (b) for the lower and upper terraces, respectively. In each panel, the energy increases from $\omega=0$ at the bottom up to $\omega=2$ on the top, with energy interval $\Delta \omega=0.05$. The arrow highlights the line with $\omega=\Delta$. For reasons to be clarified, we defined the origin of $x$ differently for the two terraces. We observe that the oscillation of LDOS on the lower terrace is
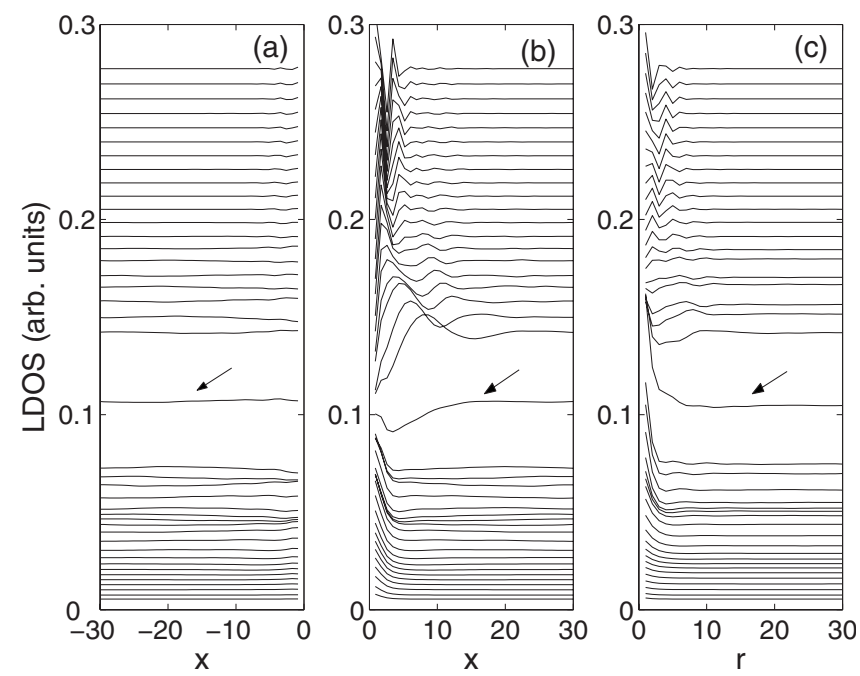

FIG. 3. LDOS as a function of the normal displacement $x$ on the (a) lower and (b) upper terraces and (c) as a function of the radial distance $r$ from a local impurity. In each panel, the energy increases from $\omega=0$ at the bottom to $\omega=2$ at the top, with energy spacing $\Delta \omega=0.05$. Arrows indicate the lines at $\omega=1$ which coincides with the bulk gap.

much weaker than that on the upper terrace. This important asymmetry can be checked experimentally and can be understood as follows. As far as the LDOS (or the local Green's function) is concerned, the upper terrace can be obtained by setting $m \rightarrow \infty$ on the left half of a surface. Therefore, the upper terrace effectively experiences a hard wall on the left. On the other hand, the lower terrace can be obtained by coupling a surface to an isolated upper-right layer through $h_{z}$. Thus, the lower terrace effectively experiences a soft boundary on the right. The asymmetry clearly follows from the difference in the effective scattering mechanism and makes it more sensible to define the origin of $x$ as the boundary of such scattering in Figs. 3(a) and 3(b).

Let us concentrate on Fig. 3(b). Here, the LDOS oscillation is negligibly small for $\omega<\Delta$, while more and more peaks appear significant for $\omega>\Delta$. The LDOS oscillation is a manifestation of quasiparticle scattering interference, ${ }^{13}$ loosely referred to as the Friedel oscillation. ${ }^{14}$ The wavelength $\lambda_{\omega}$, as is easily extracted from the first peak, should correspond to the wave number $2 k_{\omega}$, the characteristic momentum transfer during elastic quasiparticle scattering. Therefore, $k_{\omega}=\pi / \lambda_{\omega}$. By this means, we obtain a dispersion $\omega$ vs. $k_{\omega}$, which we plot in Fig. 2(e) (open squares). It clearly traces the outer border of the bulk continuum and is therefore not related to the surface Dirac fermions. The lack of visible oscillations for $\omega<\Delta$ is in nice agreement with the experiment. ${ }^{7,8,10}$ The oscillation turns out to be more visible around a local impurity which we discuss in the next section.

\section{LOCAL IMPURITY ON THE SURFACE}

We consider a scalar impurity at the origin $(\mathbf{r}=0)$ on the surface with the potential matrix $V \mathbf{I}$, as illustrated in Fig. 1(a). (Nonscalar impurities ${ }^{15}$ can be discussed along the same line but we assume scalar ones are more likely to oc- 
$\omega=0$

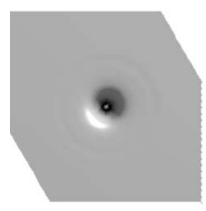

$\omega=0.6$

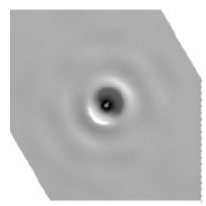

$\omega=1.2$

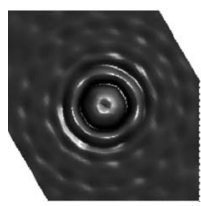

$\omega=0.2$

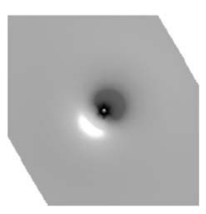

$\omega=0.8$

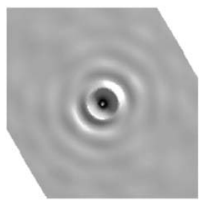

$\omega=1.4$

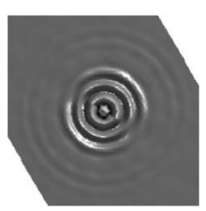

$\omega=0.4$

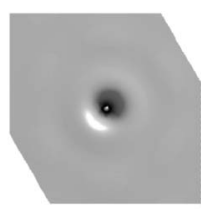

$\omega=1.0$

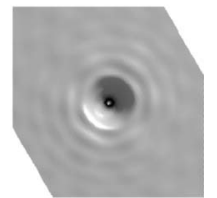

$\omega=1.6$

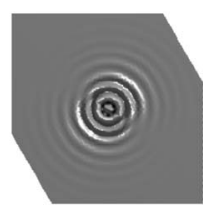

FIG. 4. Two-dimensional maps of the DOS near a local unitary impurity for specific values of $\omega$. The view field is bounded by $26 \times 26$. The strength scales with the darkness. The DOS at the origin vanishes in our case, but it is artificially reset to the value far from the impurity in order to enhance the contrast. Camera lighting is used.

cur.) Given the unperturbed surface Green's function $G$, the perturbed Green's function $\mathcal{G}$ (in real space on the surface) can be obtained again by the $T$-matrix formalism,

$$
\mathcal{G}\left(\mathbf{r}, \mathbf{r}^{\prime}\right)=G\left(\mathbf{r}-\mathbf{r}^{\prime}\right)+G(\mathbf{r}) T G\left(-\mathbf{r}^{\prime}\right)
$$

where

$$
G(\mathbf{r})=\frac{1}{M^{2}} \sum_{\mathbf{k}_{\|}} G_{\mathbf{k}_{\|}} e^{i \mathbf{k}_{\| \mid} \cdot \mathbf{r}}
$$

and $T$ is given by $T^{-1}=V^{-1} \mathbf{I}-G(0)$. The LDOS is given by

$$
\rho(\omega, \mathbf{r})=-\frac{1}{\pi} \operatorname{Im} \operatorname{Tr} \mathcal{G}(\mathbf{r}, \mathbf{r}) .
$$

We set $V \rightarrow \infty$ for a unitary impurity. The LDOS along a principle translation axis is plot in Fig. 3(c). We see LDOS oscillation for $\omega>\Delta$ similar to that in (b), although it is slightly weaker and has more complicated patterns. The oscillation below $\Delta$ is still weak but the peaks are visible for $\omega>0.5$ and they shift toward the origin with increasing energy. To have a better idea of the wavelength, we plot the LDOS map in Fig. 4 for a few values of $\omega$. The view field bounded by $26 \times 26$ is extracted from a $400 \times 400$ surface. The oscillation, even if it is present, is beyond the view field for $\omega<0.4$, while it is clear (from the dark rings) for higher energies. We extract the wave number $2 k_{\omega}$ from the radii of the rings and plot $\omega$ vs. $k_{\omega}$ in Fig. 2(e) (open circles) for $\omega$ $<\Delta$. It clearly follows the Dirac dispersion. For $\omega>\Delta$, the oscillation pattern is more complicated. In particular, there are supermodulations for $\omega=1.2$ and $\omega=1.4$, which can be understood from the double-ring structure in the corresponding map plot of the spectral function in Fig. 2(d).

\section{ANALYTICAL RESULTS FOR PURE DIRAC MODELS}

One-dimensional (1D) case: For illustrative purposes and to set up notations, we start with the single-particle Hamiltonian in a 1D Dirac model, in the momentum space, $h$ $=k \sigma_{n}$, where $\sigma_{n}$ is one of the three Pauli matrices and the Dirac velocity is set to unity henceforth. The unperturbed Matsubara Green's function in the real space is given by

$G(x)=-\int \frac{d k}{2 \pi} \frac{i \omega_{n} \sigma_{0}+k \sigma_{n}}{\omega_{n}^{2}+k^{2}} e^{i k x}=-i\left[\omega_{n} \sigma_{0}+\left|\omega_{n}\right| \operatorname{sgn}(x)\right] \phi(x)$,

where $\sigma_{0}$ is the unitary matrix, $\omega_{n}$ is the Matsubara frequency, and we defined a kernel function

$$
\phi(x)=\int \frac{d k}{2 \pi} \frac{e^{i k x}}{\omega_{n}^{2}+k^{2}}=\frac{1}{2\left|\omega_{n}\right|} \exp \left(-\left|\omega_{n} x\right|\right) .
$$

The on-site Green's function $G(0)=-i \omega_{n} \phi(0) \sigma_{0}$ turns out to be a scalar. Suppose there is a local impurity potential $V \sigma_{0}$ at the origin, the perturbed Green's function is conveniently obtained by the $T$-matrix formalism

$$
\mathcal{G}\left(x, x^{\prime}\right)=G(0)+G(x) T G\left(-x^{\prime}\right),
$$

where $T^{-1}=V^{-1} \sigma_{0}-G(0) \propto \sigma_{0}$ is the inverse of the $T$ matrix. We will concentrate on $\delta g(x)$, the trace of the change of the on-site Green's function as a function of $x$. Upon analytical continuation $i \omega_{n} \rightarrow \omega+i 0^{+}$, its imaginary part gives the change of the LDOS. For $x \neq 0$, we find

$$
\begin{aligned}
\delta g(x) & =\operatorname{Tr}[G(x) T G(-x)] \\
& \propto \operatorname{Tr}\left[\left(i \omega_{n} \sigma_{0}+i\left|\omega_{n}\right| \sigma_{n}\right)\left(i \omega_{n} \sigma_{0}-i\left|\omega_{n}\right| \sigma_{n}\right)\right] \equiv 0 .
\end{aligned}
$$

Therefore, right away from the impurity site, the LDOS is unaffected at all. Moreover, using the above $T$-matrix formalism, it is easy to verify that the off-site Green's function $\mathcal{G}\left(x, x^{\prime}\right)=-G\left(x-x^{\prime}\right)$ for $x x^{\prime}<0$ in the limit $V \rightarrow \infty$. This signifies perfect transmission (albeit with a phase lag of $\pi$ ), a manifestation of the Klein paradox. The mechanism behind this effect is the chirality (the alignment between the momentum and spin polarization) of the unperturbed eigenstates. If an incoming states were scattered backward, energy conservation requires a flipping of the spin. But a scalar impurity cannot flip the spin so the scattering matrix element between these states vanishes identically.

For comparison, the retarded Green's function in 1D metal reads $G(x) \sim-i \exp \left(i k_{\omega}|x|\right) / v_{\omega}\left(k_{\omega}\right.$ and $v_{\omega}$ are the onshell momentum and group velocity at real energy $\omega)$. The oscillation can be worked out by the $T$-matrix approach again. It has a wave number $2 k_{\omega}$ and does not decay at all in the clean limit implicitly assumed. (In reality, the oscillation must decay beyond the mean-free path.)

2D case: We write $h=\mathbf{k} \cdot \sigma=p \sigma_{x}+q \sigma_{y}$ for $2 \mathrm{D}$ Dirac fermions. We first consider a domain wall with scalar potential $V \sigma_{0}$ along the $y$ direction. Then, $q$ is still a good quantum number. The scattering geometry is illustrated in Fig. 5, where the circle of radius $k_{\omega}$ is an energy shell, radial arrows indicate the momentum-dependent spin polarization, and the 


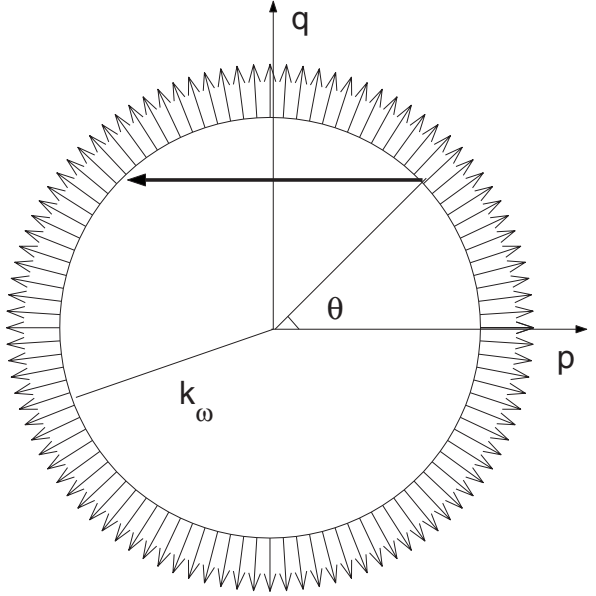

FIG. 5. Schematic plot of an energy shell at $\omega$ with an on-shell momentum $k_{\omega}$. Radial arrows indicate the spin polarizations. The long thick arrow indicates a scattering process that conserves $q$ in the case of a domain wall with its normal direction along the $p$ axis and $\theta$ is the incident angle.

long thick arrow indicate a scattering process with incident angle $\theta$ (so that $q=k_{\omega} \sin \theta$ ). The $q$-resolved unperturbed Green's function is given by

$$
\begin{aligned}
G_{q}(x) & =-\int \frac{d k}{2 \pi} \frac{i \omega_{n} \sigma_{0}+q \sigma_{y}+p \sigma_{n}}{\omega_{n}^{2}+p^{2}+q^{2}} e^{i p x} \\
& =-\left[i \omega_{n} \sigma_{0}+q \sigma_{y}+i \sqrt{q^{2}+\omega_{n}^{2}} \sigma_{x} \operatorname{sgn}(x)\right] \phi_{q}(x),
\end{aligned}
$$

where we defined

$$
\phi_{q}(x)=\frac{1}{2 \sqrt{q^{2}+\omega_{n}^{2}}} \exp \left(-\sqrt{q^{2}+\omega_{n}^{2}}|x|\right) .
$$

In particular,

$$
G_{q}(0)=-\left(i \omega_{n} \sigma_{0}+q \sigma_{y}\right) \phi_{q}(0)
$$

is no longer a scalar unless $q=0$. The $q$-resolved perturbed Green's function is given by ${ }^{12}$

$$
\mathcal{G}_{q}\left(x, x^{\prime}\right)=G_{q}\left(x-x^{\prime}\right)+G_{q}(x) T_{q} G_{q}\left(-x^{\prime}\right),
$$

with

$$
T_{q}^{-1}=V^{-1} \sigma_{0}-G_{q}(0) .
$$

By straightforward algebra, we find

$$
\delta g_{q}(x)=\operatorname{Tr}\left[G_{q}(x) T_{q} G_{q}(-x)\right]=\frac{4 q^{2} V_{q}^{-1}}{\left(i \omega_{n}+V_{q}^{-1}\right)^{2}-q^{2}} \phi_{q}(2 x),
$$

where we defined $V_{q}=V \phi_{q}(0)=V / 2 \sqrt{q^{2}+\omega_{n}^{2}}$.

Clearly, the factor of $q^{2}$ in $\delta g_{q}(x)$ follows from the requirement of spin overlap $\sin ^{2} \theta$ during elastic scattering, as illustrated in Fig. 5. This leads to $\delta g_{q}(x)=0$ for $q=0$ (normal incidence), recovering the 1D Klein paradox, as would have been anticipated. More importantly, $\delta g_{q}(x) \rightarrow 0$ in the unitary limit $V \rightarrow \infty$ (a hard wall) for all values of $q$ and consequently in the real space $\delta g(x)=\int \delta g_{q}(x) d q / 2 \pi \rightarrow 0$ as long as $|x|$ $>0$. This explains nicely the numerical results in Sec. III for the terrace that the LDOS barely oscillates below the bulk gap energy. (In Sec. III, we considered $m \rightarrow \infty$, the effect of which is however identical to $V \rightarrow \infty$ here.) In the mean time, the off-site Green's function $\mathcal{G}_{q}\left(x, x^{\prime}\right)=-G_{q}\left(x-x^{\prime}\right)$ for $x x^{\prime}$ $<0$ holds, signaling perfect transmission in analog to the 1D case. This surprising result indicates that the Klein paradox also works in 2D but only for a hard wall.

For general strength of $V$ (or soft walls), the asymptotic behavior in the limit of $\left|\omega_{n} x\right| \gg 1$ (where a saddle-point approximation is valid) is given by

$$
\begin{aligned}
\delta g(x)= & \int \frac{d q}{2 \pi} \delta g_{q}(x) \\
& \sim \frac{V}{\left(2+i V \operatorname{sgn} \omega_{n}\right)^{2} \pi|x|} \sqrt{\frac{\pi}{\left|\omega_{n} x\right|}} \exp \left(-2\left|\omega_{n} x\right|\right),
\end{aligned}
$$

where $q=k_{\omega} \sin \theta$ is used. Upon analytical continuation $\left|\omega_{n}\right| \rightarrow i \omega$, this amounts to an oscillation of LDOS with an energy-dependent wave number $2 k_{\omega}=2|\omega|$ and an envelope function that decays as $|x|^{-3 / 2}$.

For comparison, in a 2D metal, $G_{q}(x) \sim$ $-i \exp \left(i k_{\omega}|x| \cos \theta\right) / v_{\omega} \cos \theta$ in real frequency. By the $T$-matrix formalism, the domain wall leads to $\delta g(x) \propto$ $-\int d \theta \exp \left(2 i k_{\omega}|x| \cos \theta\right) /\left(V^{-1} v_{\omega} \cos \theta+i\right)$

$\propto \exp \left(2 i k_{\omega}|x|\right) / \sqrt{k_{\omega}|x|}$ for $k_{\omega}|x| \gg 1$. So the LDOS oscillation decays as $\left|k_{\omega} x\right|^{-1 / 2}$ near a domain wall in a 2D metal. ${ }^{16}$ Notice that $k_{\omega=0}$ is just the Fermi wave number.

For a point impurity in 2D, the unperturbed Green's function for Dirac fermions is given by

$$
\begin{aligned}
G(\mathbf{r}) & =-\int \frac{d^{2} k}{(2 \pi)^{2}} \frac{i \omega_{n} \sigma_{0}+\mathbf{k} \cdot \sigma}{\omega_{n}^{2}+k^{2}} e^{i \mathbf{k} \cdot \mathbf{r}} \\
& =-i\left[\omega_{n} \sigma_{0}+\left(\left|\omega_{n}\right|+\frac{1}{2 r}\right) \hat{\mathbf{r}} \cdot \sigma\right] \phi(r),
\end{aligned}
$$

where we find

$$
\phi(r) \sim \frac{\exp \left(-\left|\omega_{n}\right| r\right)}{4 \pi} \sqrt{\frac{2 \pi}{\left|\omega_{n}\right| r}}
$$

in the asymptotic limit $\left|\omega_{n}\right| r \gg 1$. For the on-site Green's function, a cutoff $\Lambda$ in momentum has to be introduced so that

$$
G(0)=\frac{-i \omega_{n} \sigma_{0}}{4 \pi} \ln \frac{\Lambda^{2}}{\omega_{n}^{2}} .
$$

Using the $T$-matrix formalism with $T^{-1}=V^{-1} \sigma_{0}-G(0)$, we find

$$
\begin{aligned}
\delta g(\mathbf{r}) & =\operatorname{Tr} G(\mathbf{r}) T G(-\mathbf{r}) \\
& \sim \frac{1}{4 \pi V^{-1}+i \omega_{n} \ln \left(\Lambda^{2} / \omega_{n}^{2}\right)} \frac{\exp \left(-2\left|\omega_{n}\right| r\right)}{r^{2}}
\end{aligned}
$$

to leading order in $1 /\left|\omega_{n}\right| r$. Upon analytical continuation, we see that the change of LDOS oscillates with an energy- 
dependent wave number $2 k_{\omega}=2|\omega|$ and decays as $r^{-2}$. Notice that the oscillation exists even for $V \rightarrow \infty$, in contrast to the case of the domain wall. This is consistent with the results in Sec. IV. However, there is an important difference. Here, a sharp resonance state appears at $\omega=0$ (in the unitary limit $V \rightarrow \infty)$ as seen from analytical continuation of $\delta g(\mathbf{r})$ : a wellknown result in many contexts. ${ }^{17}$ This resonance state is absent in Sec. IV, as can be seen in Fig. 3(b) or 4. Qualitatively, this is due to the fact that surface Dirac fermions are not completely confined on the surface. Depending on the planar momentum, the wave function has varying extent of amplitude on the surface. Therefore, the impurity is only partially seen by the surface Dirac fermions. Moreover, they exist only in a limited regime in the momentum space. An accurate discussion of these issues is left for future studies.

For comparison, in a 2D metal, $G(\mathbf{r})=$ $-i \int d \theta \exp \left(i k_{\omega} r \cos \theta\right) k_{w} / 2 \pi v_{\omega} \propto \exp \left(i k_{w} r\right)\left(k_{\omega} r\right)^{-1 / 2}$ for $k_{\omega} r$ $\gg 1$. By the $T$-matrix approach, a local impurity leads to $\delta g(\mathbf{r}) \propto G(\mathbf{r})^{2} \propto \exp \left(2 i k_{\omega} r\right) / k_{\omega} r$ for $k_{\omega} r \gg 1$. So the LDOS oscillation decays as $r^{-1}$ near a local impurity in a $2 \mathrm{D}$ metal. ${ }^{16}$

Similar analysis could be proceeded for 3D Dirac fermions in the presence of two-, one-, and zero-dimensional impurities. As the impurity states would not be easily probed by STM, we do not go into detailed analysis. Some interesting behaviors are as follows. First, a 2D hard wall does not lead to any oscillation in LDOS away from the wall. For lowerdimension or general scalar impurities, the oscillation is present and decays faster than that for normal metals by a factor of $1 / r$.

\section{SUMMARY AND REMARKS}

We analyze the behavior of surface states of topological insulators against local and extended impurities. Using an effective 3D lattice model, we first obtain the spectral function of the surface states. This enables us to identify the contributions from massless Dirac fermions and bulk continuum. We then study the effects of a local impurity and a terrace on the surface. Away from a local impurity, the spatial oscillation in LDOS below the bulk gap $\Delta$ is much weaker than that above $\Delta$. The LDOS oscillation is globally weak on the lower terrace, while on the upper terrace, the LDOS oscillation is barely visible below $\Delta$, in perfect agreement with the STM measurement, but it is significant above $\Delta$. The asymmetry of the LDOS oscillation on the lower and upper terraces is attributed to the difference in the effective scattering mechanism. From the LDOS oscillations, we extract the modulation wave number $2 k_{\omega}$ as a function of energy $\omega$. The dispersion ( $\omega$ vs. $k_{\omega}$ ) follows that of Dirac fermions for $\omega<\Delta$, but it follows the border of the bulk continuum for $\omega>\Delta$. The numerical results combine to reveal that the surface Dirac fermions are rather immune to the imperfections. We discuss such a behavior analytically by pure Dirac models. Because of a cancellation due to the alignment of momentum and spin polarization, the $2 k_{\omega}$ oscillation in LDOS, if present, decays asymptotically faster by a factor of $1 / r$ than that for usual fermions. Specifically, the oscillation is absent for a hard domain wall. Such behaviors are consistent with the 3D lattice model. We also find and discuss differences between surface and pure Dirac fermions.

Before closing, some remarks are in order. The simple lattice model studied here captures the intrinsic properties of the surface Dirac fermions. Material-dependent fine features would require modification of the model. For example, in order to capture the sixfold symmetric wavy energy shells (in the momentum space) for surface Dirac fermions, ${ }^{4}$ which is important for quasiparticle scatting to pick out specific momentum transfer as discussed elsewhere, ${ }^{18}$ a warping term ${ }^{19}$ has to be included. This can be done in the lattice model by adding an in-plane hopping term $2 i t_{w} \Gamma_{4} \Sigma_{b} f_{b} \sin k_{b}$ in $h_{\mathbf{k}_{\|}}^{2 d}$, where $t_{w}$ is the hopping amplitude, $\Gamma_{4}$ is a Dirac matrix in the same Clifford algebra that includes $\Gamma_{0,1,2,3}$ already defined, and $f_{b}=1,-1,1$ for the three independent nearest-neighbor bonds $b$. This term has $f$-wave symmetry and leads to elongation of the surface-state energy shells in the $\Gamma-M$ directions. However, our preliminary calculations show that in order to get warping features comparable to experiments, we need a very large hopping amplitude $2 t_{w} \gg 1$ (in units defined above). This seems very unlikely in reality and calls for a realistic model based on first-principles calculations. Investigations in this direction are under consideration. Second, we notice that a real terrace edge is not likely a straight line. This may be mapped to a straight edge but with excess impurities nearby. According to our results of both local impurity and terrace, a wiggling terrace edge should also lead to visible oscillations below the bulk gap, consistent with experiments. ${ }^{9}$ On the other hand, in a model with multiple Dirac cones for the surface states, elastic scattering between cones is not suppressed by the chirality and is therefore expected to induce visible LDOS oscillations even below the bulk gap.

Note added. While finalizing this writing, we became aware of related works using a 2D continuum model with warping terms. ${ }^{19}$

\section{ACKNOWLEDGMENTS}

The work in Nanjing was supported by NSFC Grants No. 10974086 and No. 10734120, the Ministry of Science and Technology of China (under the Grants No. 2006CB921802 and No. 2006CB601002), and the 111 Project (under the Grant No. B07026). The work in Hong Kong was supported by Hong Kong's RGC grant HKU 10/CRF/08. 
*qhwang@nju.edu.cn

${ }^{1}$ L. Fu and C. L. Kane, Phys. Rev. B 76, 045302 (2007).

${ }^{2}$ X. L. Qi, T. L. Hughes, and S. C. Zhang, Phys. Rev. B 78, 195424 (2008); X. L. Qi, T. L. Hughes, S. Raghu, and S. C. Zhang, Phys. Rev. Lett. 102, 187001 (2009).

${ }^{3}$ L. Fu, C. L. Kane, and E. J. Mele, Phys. Rev. Lett. 98, 106803 (2007); J. E. Moore and L. Balents, Phys. Rev. B 75, 121306(R) (2007); R. Roy, ibid. 79, 195321 (2009).

${ }^{4}$ Y. L. Chen, J. G. Analytis, J.-H. Chu, Z. K. Liu, S.-K. Mo, X. L. Qi, H. J. Zhang, D. H. Lu, X. Dai, Z. Fang, S. C. Zhang, I. R. Fisher, Z. Hussain, and Z.-X. Shen, Science 325, 178 (2009); D. Hsieh, Y. Xia, L. Wray, D. Qian, A. Pal, J. H. Dil, J. Osterwalder, F. Meier, G. Bihlmayer, C. L. Kane, Y. S. Hor, R. J. Cava, and M. Z. Hasan, ibid. 323, 919 (2009); D. Hsieh, D. Qian, L. Wray, Y. Xia, Y. S. Hor, R. J. Cava, and M. Z. Hasan, Nature (London) 452, 970 (2008); H. J. Noh, H. Koh, S. J. Oh, J. H. Park, H. D. Kim, J. D. Rameau, T. Valla, T. E. Kidd, P. D. Johnson, Y. Hu, and Q. Li, Europhys. Lett. 81, 57006 (2008); Y. Xia, L. Wray, D. Qian, D. Hsieh, A. Pal, H. Lin, A. Bansil, D. Grauer, Y. S. Hor, R. J. Cava, and M. Z. Hasan, Nat. Phys. 5, 398 (2009).

${ }^{5}$ H. Zhang, C.-X. Liu, X.-L. Qi, X. Dai, Z. Fang, and S.-C. Zhang, Nat. Phys. 5, 438 (2009).

${ }^{6}$ X. L. Qi, R. D. Li, J. Zang, and S. C. Zhang, Science 323, 1184 (2009).

${ }^{7}$ P. Roushan, J. Seo, C. V. Parker, Y. S. Hor, D. Hsieh, D. Qian, A. Richardella, M. Z. Hasan, R. J. Cava, and A. Yazdani, Nature (London) 460, 1106 (2009).

${ }^{8}$ Z. Alpichshev, J. G. Analytis, J. H. Chu, I. R. Fisher, Y. L. Chen, Z. X. Shen, A. Fang, and A. Kapitulnik, arXiv:0908.0371 (unpublished).
${ }^{9}$ T. Zhang, P. Cheng, X. Chen, J.-F. Jia, X. Ma, K. He, L. Wang, H. Zhang, X. Dai, Z. Fang, X. Xie, and Q.-K. Xue, arXiv:0908.4136 (unpublished).

${ }^{10}$ K. K. Gomes, W. Ko, W. Mar, Y. Chen, Z.-X. Shen, and H. C. Manoharan, arXiv:0909.0921 (unpublished).

${ }^{11}$ Y. Zhang, K. He, C.-Z. Chang, C.-L. Song, L. Wang, X. Chen, J. Jia, Z. Fang, X. Dai, W.-Y. Shan, S.-Q. Shen, Q. Niu, X. Qi, S.-C. Zhang, X. Ma, and Q.-K. Xue, arXiv:0911.3706 (unpublished).

${ }^{12}$ Q.-H. Wang and Z. D. Wang, Phys. Rev. B 69, 092502 (2004).

${ }^{13}$ Q.-H. Wang and D.-H. Lee, Phys. Rev. B 67, 020511(R) (2003); J. E. Hoffman, K. McElroy, D.-H. Lee, K. M. Lang, H. Eisaki, S. Uchida, and J. C. Davis, Science 297, 1148 (2002); K. McElroy, R. W. Simmonds, J. E. Hoffman, D.-H. Lee, J. Orenstein, H. Eisaki, S. Uchida, and J. C. Davis, Nature (London) 422, 592 (2003).

${ }^{14}$ J. Friedel, Nuovo Cimento, Suppl. 7, 287 (1958).

${ }^{15}$ Q. Liu, C. X. Liu, C. Xu, X. L. Qi, and S. C. Zhang, Phys. Rev. Lett. 102, 156603 (2009).

${ }^{16}$ M. F. Crommie, C. P. Lutz, and M. Eigler, Nature (London) 363, 524 (1993).

${ }^{17}$ M. I. Salkola, A. V. Balatsky, and D. J. Scalapino, Phys. Rev. Lett. 77, 1841 (1996); Q.-H. Wang, ibid. 88, 057002 (2002); V. M. Pereira, F. Guinea, J. M. B. Lopes dos Santos, N. M. R. Peres, and A. H. Castro Neto, ibid. 96, 036801 (2006).

${ }^{18}$ X. Zhou, C. Fang, W.-F. Tsai, and J. P. Hu, Phys. Rev. B 80, 245317 (2009).; W.-C. Lee, C. Wu, D. P. Arovas, and S.-C. Zhang, ibid. 80, 245439 (2009).

${ }^{19}$ L. Fu, Phys. Rev. Lett. 103, 266801 (2009). 